\title{
Convergence, electrostatic potential, and density measurements in a spherically convergent ion focus
}

\author{
T. A. Thorson, ${ }^{\text {a) }}$ R. D. Durst, R. J. Fonck, and L. P. Wainwright \\ University of Wisconsin-Madison, 1500 Engineering Drive, Madison, Wisconsin 53706
}

(Received 18 June 1996; accepted 11 October 1996)

\begin{abstract}
Unique measurements of the basic plasma-flow characteristics in a low pressure $\left(\leqslant 53 \mathrm{mPa} \mathrm{H}_{2}\right)$ spherically convergent ion focus are obtained using high-voltage $(\leqslant 5 \mathrm{kV})$ emissive and double probes. The radial plasma potential distribution agrees with a collisionless, recirculating, space-charge-limited current model. Flow convergence increases with voltage and neutral pressure and decreases with cathode grid wire spacing and current. Core radii within 4-5 times the ideal geometric limit are measured, and the observed core sizes are consistent with predictions from a multipass orbit model which includes asymmetries in the accelerating potential well. A virtual anode is observed in the converged core region, and no evidence for multiple potential well structures in the core is found. Measurements of the core ion density $\left(n_{\mathrm{ic}} \sim 10^{15} \mathrm{~m}^{-3}\right)$ are consistent with simple flow convergence models. (c) 1997 American Institute of Physics.
\end{abstract} [S1070-664X(97)02701-8]

\section{INTRODUCTION}

The spherically convergent ion focus (SCIF) is an alternative plasma confinement scheme in which ions are electrostatically confined, accelerated, and concentrated at fusionrelevant energies. Ions introduced at the edge of the system fall into a spherically symmetric potential well formed by a highly transparent cathode, and a condensed core, consisting of monoenergetic ions, forms in the center of the device. Individual ions oscillate in the electrostatic well, and a recirculating, non-Maxwellian flow results. Fusion reactions then occur from high-energy collisions between the counterstreaming ions or with background neutral particles.

While many others have done experimental studies on these types of devices, they have concentrated mainly on fusion reactivity measurements with relatively high neutral pressure glow discharges $(>130 \mathrm{mPa}) .{ }^{1-3}$ In contrast, the Wisconsin Spherically Convergent Ion Focus (WISCIF) experiments concentrate on determining the basic plasma characteristics of the ion flow for lower pressure $(<53 \mathrm{mPa})$, driven (non-self-sustaining) discharges in hydrogen. In this regime, the ion flow is essentially collisionless with respect to neutral particle interactions. The first direct measurements of the central ion density, radial electrostatic potential profile, and accelerating potential asymmetries are also provided using high-voltage electrostatic probes.

Potential advantages of a SCIF-driven system are that energetic ions are easily obtained, the cathode potential may be tuned in energy to the peak cross section for many different fusion reactions (i.e., D-T, D- ${ }^{3} \mathrm{He}, p-{ }^{11} \mathrm{~B}$, and $p-{ }^{7} \mathrm{Li}$ ), and the fuel inventory is lower than conventional particle sources. SCIF devices can then produce high-energy neutrons, protons, alphas, etc., and they may make attractive

${ }^{a)}$ Electronic mail: thorson@uwmfe.neep.wisc.edu sources $\left(10^{6}-10^{12}\right.$ particles/s) suitable for neutron activation or scattering analysis, oil well logging, and detection of chemical weapons or explosives. ${ }^{4}$ However, the higher range of these required fusion reaction rates exceeds those achieved in SCIF devices to date $\left(<10^{8} \mathrm{n} / \mathrm{s} \mathrm{D}-\mathrm{D},<10^{10} \mathrm{n} / \mathrm{s}\right.$ D-T). ${ }^{1-3,5}$

The SCIF concept was originally conceived in the $1950 \mathrm{~s},{ }^{6,7}$ and experiments started in the 1960s using material grid electrodes to provide the accelerating potentials. ${ }^{1}$ Early fusion reactivity measurements indicated higher-thanexpected neutron production, and work continued through the early 1970s until power balance estimates showed that these gridded-cathode systems could not extrapolate to a power production facility. ${ }^{7}$ Recently, new theoretical concepts and analyses, ${ }^{8-10}$ potential engineering and medical applications, ${ }^{4,8}$ plus a renewed interest in alternative concepts for fusion power production have all rekindled discussion of SCIF devices.

In addition to gridded systems, two other approaches that generate virtual cathodes and avoid the power and stress-loading problems with grids are currently under study. One is the Polywell ${ }^{\mathrm{TM}}$ concept, wherein a polyhedral magnetic cusp configuration confines high-energy electron beams that form a perfectly transparent virtual cathode. ${ }^{8,9}$ The second approach confines high-energy electrons in a Penning or similar trap, which results in a spherically symmetric potential well for ions. ${ }^{10}$ While these gridless devices have more promise for higher reactivity, recent theoretical appraisals of such systems, which more extensively account for thermalizing collisional effects and the overall power balance, determine that a power production scheme is not possible based on these types of devices without circumventing these basic issues. ${ }^{11,12}$

Despite the differences in the origin of the confining electrostatic fields, a common figure of merit for all proposed 
SCIF approaches is the attainable degree of focusing of the ion flow at the center. This is known as the flow convergence, and it is a critical parameter for all high-reactivity designs which rely on the counter-streaming ion flows providing the system reactivity. Physical processes that may limit or determine the flow convergence include the structure of the electrostatic well in the presence of the ion flow, the magnitude of asymmetries in the confining potential, and scattering collisions with background neutrals or counterstreaming ions.

The presence of any virtual anode structure, due to excess ion accumulation at $r \approx 0$, can perturb the ion flow and convergence as well as change global collisional effects through changes in the ion energies locally at $r \approx 0$. In addition, this anode would presumably trap electrons, and some investigators, using one-dimensional theoretical models, suggest that a secondary virtual cathode may form inside the virtual anode. ${ }^{1,2,13,14}$ It is further speculated that an energetic, recirculating population of ions could be trapped in this virtual cathode, which may explain the high reactivity performance of the early experiments. However, no conclusive evidence of such core potential structures has been observed experimentally, and models that relax the perfectly radial, collisionless assumption do not predict the multiple-well formation. ${ }^{15-17}$

Potential well asymmetries are usually defocusing and hence would be expected to degrade the spherical ion flow. This, in turn, leads to a more diffuse central ion core region with a correspondingly reduced fusion reactivity for SCIF devices that rely on reactions between the counter-streaming ion flows (beam-beam dominated).

The convergence to a high density core near $r=0$ can also be significantly influenced by collisional perturbations of the recirculating ion orbits in the potential well. Nevins ${ }^{11}$ and Rider ${ }^{12}$ predict that thermalization of ion flows through core collisions would lead to the collapse of the convergence in an idealized highly recycling central ion flow. Although the gridded experiments studied to date find a steady flow convergence, the particles are removed at a rate faster than the thermalization rate (i.e., via grid or charge-exchange collisions, see Sec. II).

In spite of these arguments, there is little or no past experimental data on the generic behavior of the ion flow and flow convergence in SCIF devices. The purpose of the present work is to provide such critical data, which will, in turn, improve the fundamental understanding for all types of these systems. A flexible, gridded-electrode scheme is chosen for these experiments due to its relatively simple and inexpensive design, and electrostatic probes and visible imaging diagnostics are used to characterize the ion flow.

This work concentrates on relatively low density, lower energy $\left(n \sim 10^{16} \mathrm{~m}^{-3}, E_{i} \approx 5-20 \mathrm{keV}\right)$ SCIF operation, where previous experiments indicate significant fusion reactivity. ${ }^{1-3}$ Lower neutral pressure $\left(\begin{array}{llll}\leqslant 53 & \mathrm{mPa} & \mathrm{H}_{2}\end{array}\right)$, "converged-core" ion flows are studied in order to minimize the effects of neutral collisions on the flow convergence. The resulting discharge characteristics are also different than the glow discharge modes studied previously, and unique mea-

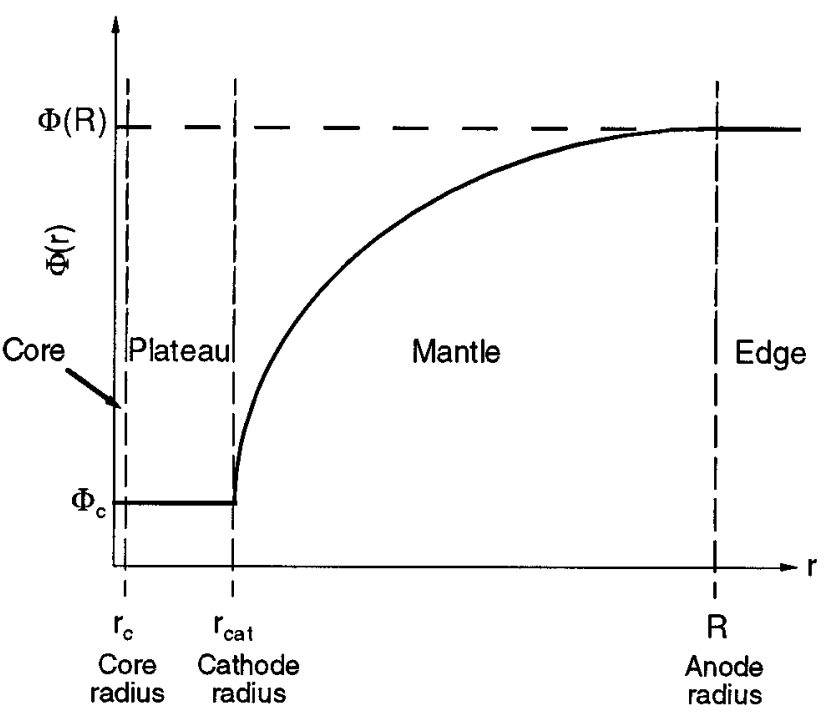

FIG. 1. Definitions for the four regions of interest in a SCIF device.

surements of the flow convergence, electrostatic potential, and ion density are presented here.

\section{ELEMENTARY ION FLOW CONVERGENCE MODEL}

Understanding the behavior of the ion density and velocity distributions is critical for estimating or extrapolating the fusion reactivity of any SCIF system. To lowest order, these distributions can be approximated using an elementary collisionless ion orbit model where only radial dependencies are considered. Any perpendicular velocity of an ion is included in this model only in conserving angular momentum, and hence the radial velocity of the flowing ions is directly related to the local electrostatic potential. The ion density distribution is then determined by conserving the total ion current in the device.

The actual situation is, of course, much more involved. The local ion flow, electron distribution, and the plasma potential distribution all interact and adjust themselves accordingly to achieve a self-consistent equilibrium. This leads to deviations of the plasma potential distribution from the imposed vacuum field. For example, excess ion charge in the core region can lead to a virtual anode region there and cause scattering of the incident ions. A full description of the system in dynamic equilibrium awaits solution of the FokkerPlanck equation in the fully three-dimensional environment. However, the simple ballistic model discussed here is traditionally taken to provide a lowest-order description of the system, and it can be employed to indicate the expected scalings of critical parameters with system properties and thus provide a starting point for comparison with experiment.

In order to justify the collisionless assumption, a comparison of the various ion collision rates is needed. For the ion densities and neutral pressures studied in the WISCIF device (see Sec. IV below), the ion-neutral collision rate $\left(\sim 10^{5} / \mathrm{s}\right)$ is lower than the effective ion-cathode collision rate $\left(\approx 2 \times 10^{5} / \mathrm{s}\right) .{ }^{18}$ The ion-ion collision rate is $\sim 10^{-3} / \mathrm{s}$ for the counter-flowing ions and $\sim 10^{4} / \mathrm{s}$ for the co-propagating ions, and they are therefore less significant than the ion- 


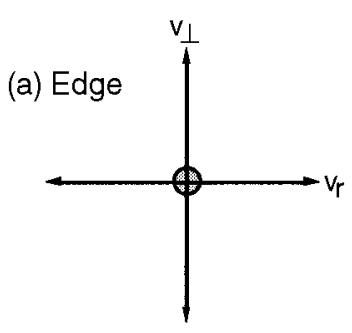

(b)
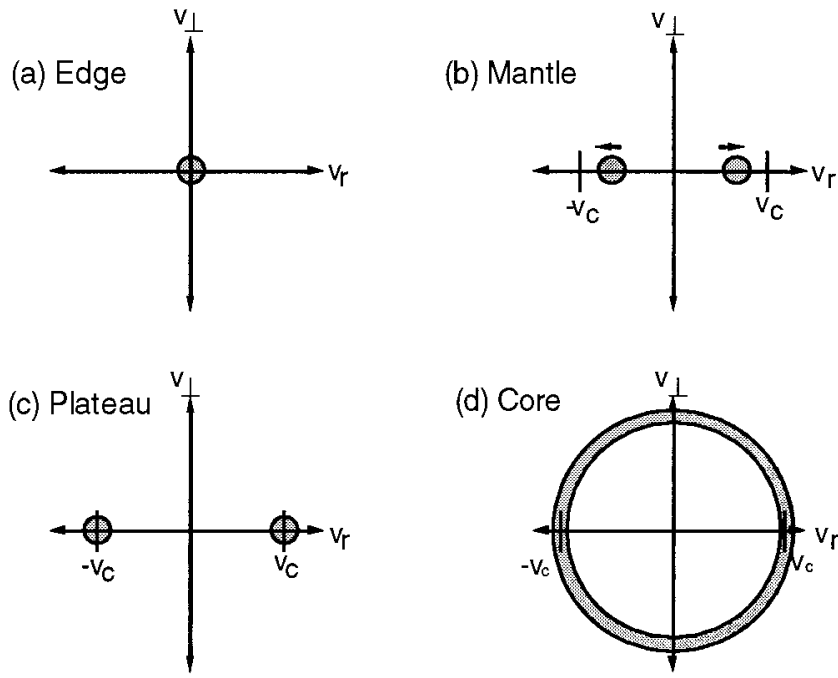

FIG. 2. Qualitative behavior of the ideal background ion velocity distributions in the lab frame for the different regions of interest (not to scale)-(a) edge: cold, thermal at $\langle v\rangle=0$, (b) mantle: low $T_{i}, v_{r}<v_{c}$, (c) plateau: $v_{r}= \pm v_{c},(\mathrm{~d})$ core: isotropic shell $\left|v_{r}\right|=v_{c}$. In all cases, $T_{i} \ll \Phi_{c}$.

cathode and ion-neutral collisions. ${ }^{19}$ Since the ion lifetime is limited mainly by the transparency of the cathode, the ions essentially appear as a collisionless species until termination at the cathode.

The ion flow characteristics change throughout the SCIF system, and it is helpful to distinguish them for different regions (see Fig. 1)

$$
\begin{aligned}
& r>R \rightarrow \text { edge }, \\
& r_{\text {cat }}<r \leqslant R \rightarrow \text { mantle, } \\
& r_{c}<r \leqslant r_{\text {cat }} \rightarrow \text { plateau, } \\
& r \leqslant r_{c} \rightarrow \text { core }
\end{aligned}
$$

where $R$ is the anode radius, $r_{\text {cat }}$ is the cathode radius, and $r_{c}$ is the core radius.

Figure 2 indicates the idealized background ion distributions for the various zones identified above. For the low pressure discharges, a cold plasma is generated at the edge, and any ions moving toward the cathode are accelerated to full energy in the mantle region. The plateau zone ideally contains monoenergetic ions inside the cathode, and the core distribution consists of a thin, spherical shell in velocity space at a radius of the core velocity.

Angular momentum of the ions at the edge, due to their perpendicular thermal velocity, $v_{\perp}(R)$, will keep the flow from perfectly converging to $r=0$, but to lowest order one can assume the flow is converging to a radius $r_{c}$, defined as the core radius. All ions are assumed to be noninteracting and flow through the core with a velocity $v_{c}=\sqrt{2 q \Phi_{c} / M}$, where $\Phi_{c}$ is the electric potential in the core [for $\Phi(R)=0$ ], $q$ is the ion charge, and $M$ is the ion mass. By setting the average angular momentum of the ions at the edge equal to that of the ions passing through the core, $M\left\langle v_{\perp}\right\rangle R \approx(2 / 3) M v_{c} r_{c}$, the core radius is then given by

$$
r_{c}=\frac{3}{2} R \frac{\left\langle v_{\perp}\right\rangle}{v_{c}} \approx \frac{3}{2} R \sqrt{\frac{k T_{\perp i}(R)}{q \Phi_{c}}},
$$

where $T_{\perp i}(R)$ is the edge perpendicular ion temperature. Therefore, $r_{c}$ should scale as the inverse square root of $\Phi_{c}$.

Assuming no sources or sinks of particles, total current conservation demands that $n_{i}(r) v(r) r^{2}=$ const for all $r$, where $n_{i}(r)$ is the local ion density. The expected steadystate radial profile is then

$$
n_{i}(r)=n_{i}(R)\left(\frac{R}{r}\right)^{2} \frac{v(R)}{v(r)}=n_{i}(R)\left(\frac{R}{r}\right)^{2} \sqrt{\frac{E_{\|}(R)}{q \Phi(r)}},
$$

where $E_{\|}(R)$ is the radial energy of the ions at the edge.

Angular momentum conservation also prevents the ion density from diverging at $r=0$, and a constant density is invoked in the core to provide a lowest-order estimate. Since the effective core area is a circle of radius $r_{c}$, the core density estimate becomes

$$
n_{\mathrm{ic}}=4 n_{i}(R)\left(\frac{R}{r_{c}}\right)^{2} \frac{v(R)}{v_{c}}=4 n_{i}(R)\left(\frac{R}{r_{c}}\right)^{2} \sqrt{\frac{E_{\|}(R)}{q \Phi_{c}}} .
$$

This model conserves average angular momentum and total particle flux, and while it fails to keep the density continuous at $r_{c}$, Eq. (3) should provide reasonable scaling estimates for comparisons with the experimental results.

By substituting for $r_{c}$ from Eq. (1), an estimate for the convergence factor (ratio of core to edge density) is found from Eq. (3)

$$
\frac{n_{\mathrm{ic}}}{n_{i}(R)}=\frac{16}{9} \frac{\sqrt{E_{\|}(R)}}{k T_{\perp i}(R)} \sqrt{q \Phi_{c}} .
$$

Therefore, the core density should optimally scale as the square root of the core potential for given edge plasma conditions.

The core density and system reactivity will ultimately depend on how much recirculating ion current can be sustained, and the resulting ion space-charge limits the magnitude of this current for steady-state systems. The ideal spacecharge-limited ion current between concentric spheres (mantle region) is

$$
I_{\operatorname{man}}=\frac{16 \pi \epsilon_{0}}{9} \sqrt{\frac{2 q}{M}} \frac{V^{3 / 2}}{\alpha^{2}},
$$

where $\epsilon_{0}$ is the permittivity of free space, $V$ is the voltage difference between the spheres, and $\alpha$ is a geometrical factor that is related to the radii of the spherical electrodes ${ }^{20}$ $[\alpha \sim \ln (r / R)]$. Assuming no sources or sinks of particles between the electrodes (consistent at low pressure and density), Eq. (5) can be solved for the voltage

$$
V(r)=\left[\frac{9 I_{\operatorname{man}} \alpha^{2}(r)}{16 \pi \epsilon_{0}} \sqrt{\frac{M}{2 q}}\right]^{2 / 3},
$$

where $\alpha$ determines the radial dependence $(\alpha \rightarrow 0$ as $r \rightarrow R)$. This equation, derived originally for solid electrodes, also holds for semitransparent grids with counter-streaming ion flows if $I_{\text {man }}$ represents the total recirculating ion current between the spheres. 


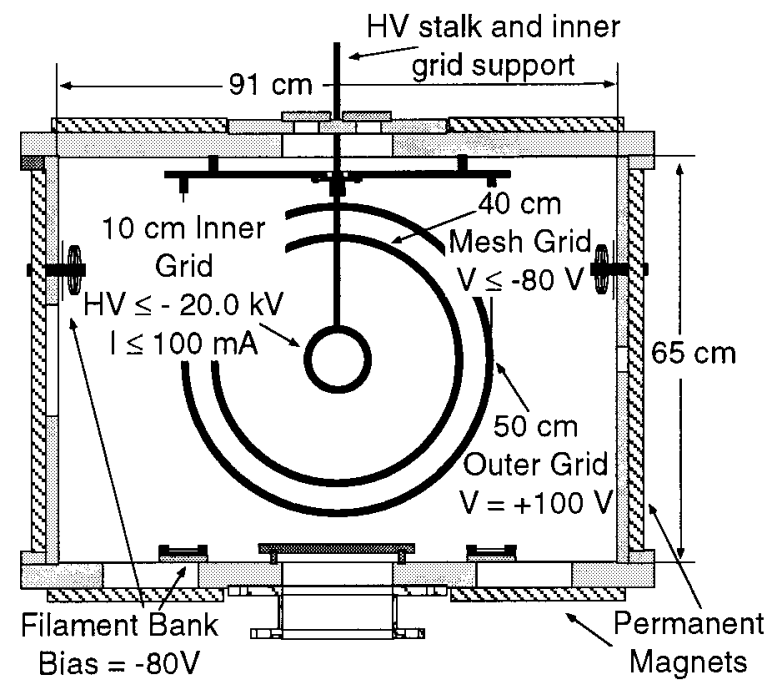

FIG. 3. Schematic of the WISCIF device including typical operating voltages. The grid dimensions listed in the figure are diameters.

For a gridded system, the amount of recirculating current is mainly limited by the effective cathode transparency. Assuming that the ions are equally likely to be lost at each pass through the cathode, the recirculation density enhancement factor, $\xi$, is given by ${ }^{21}$

$$
\xi \equiv \frac{I_{\text {man }}}{I_{\text {cat }}}=\frac{1}{\left(1-\eta^{2}\right)},
$$

where $I_{\text {cat }}$ is the ion current collected by the cathode and $\eta$ is the geometric grid transparency $\left[\eta \equiv\left(\right.\right.$ open area) $\left./ 4 \pi r_{\text {cat }}^{2}\right]$.

If the number of secondary electrons emitted from the grid due to ion impact is given by $\delta$, then the measured ion grid current $\left(I_{\text {meas }}\right)$ is $(1+\delta) I_{\text {cat }}$, and the recirculation factor relates to the measured grid current by

$$
\xi=\frac{I_{\text {man }}(1+\delta)}{I_{\text {meas }}} .
$$

By comparing the measured radial electrostatic potential distribution, $V(r)$, with Eq. (6), the total recirculating current between the spheres, $I_{\text {man }}$, can be determined, and then an estimate for $\xi$ is given by Eq. (8) using the measured grid current.

\section{EXPERIMENTAL APPARATUS}

The Wisconsin Spherically Convergent Ion Focus (WISCIF) experimental device, shown in Fig. 3, consists of three concentric, stainless-steel wire globes housed in a cylindrical vacuum vessel. An extended plasma, formed about the outermost grid, provides an ion source localized at large radii. Hot tungsten filaments $(0.01 \mathrm{~cm}$ diameter, $5 \mathrm{~cm}$ long, biased at $-80 \mathrm{~V})$ generate the primary electrons which accelerate towards the positively biased outer grid $(+100 \mathrm{~V})$ to collisionally ionize the background gas (typically $\mathrm{H}_{2}$ ). The negatively biased middle grid $(-80 \mathrm{~V})$ shields these primary electrons away from the flow region and localizes the edge plasma to radii greater than $20 \mathrm{~cm}$. A fine mesh with a spacing $(0.04 \mathrm{~cm})$ less than the local electron Debye length $(\sim 0.1$

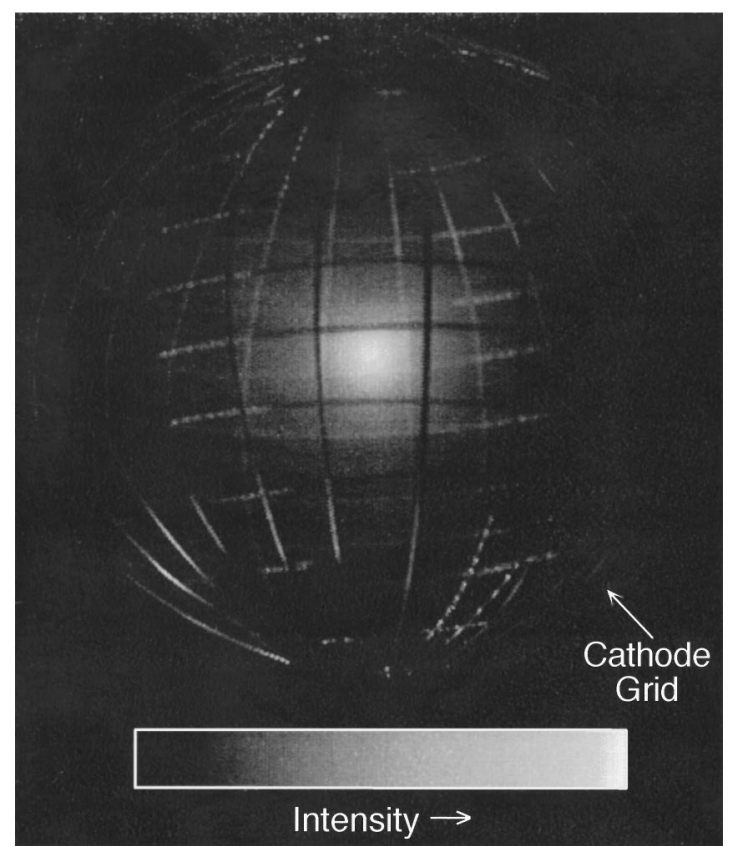

FIG. 4. A CCD camera image of the $\mathrm{H}_{\alpha}$ emission for a $17 \mathrm{kV}, 80 \mathrm{~mA}$ converged core mode showing a $0.6 \mathrm{~cm}$ radius core. The grid shown is a 5.0 $\mathrm{cm}$ radius cathode grid with a $15^{\circ}$ poloidal grid spacing.

$\mathrm{cm})$ is placed on this grid to provide an electrostatic barrier for the electrons, and permanent magnets are arranged around the outer wall to enhance the primary electron lifetime. The innermost cathode grid $\left(r_{\text {cat }}=5 \mathrm{~cm}\right)$ provides the deep, accelerating potential well $(5-17 \mathrm{kV})$ for the ion flow. The collected cathode current $(20-80 \mathrm{~mA})$ is controlled by varying the edge plasma density with the primary electron source. Ion flows were generated and sustained in this manner for pressures as low as $13 \mathrm{mPa}$ in hydrogen. While these operating conditions are in no way optimized for fusion reactivity production, ion flows of this energy region do provide a suitable plasma to study the basic ion flow physics.

A spectrally filtered charge-coupled-device (CCD) is used to image the core region and provide core size estimates under variations of cathode currents, voltages, potential asymmetries, and background pressures. A tightly focused, converged-core region is readily evident near the origin of the high-voltage cathode (see Fig. 4). The size and brightness of this high-energy core varies considerably with experimental parameters, but its existence is generally robust in that it is readily attained and persists stably. While the recorded emission is likely dominated by electron-impact excitation of the background gas, the behavior of the core reflected in these images is consistent with the probe measurements in the ion core region to lowest order.

Electrostatic probes are used to characterize the plasma and ion flow in different regions of the device. A standard Langmuir probe provides the edge plasma density and electron temperature, and double or emissive probes mounted an alumina-Pyrex probe shaft for $30 \mathrm{kV}$ isolation allow measurements inside the flow and core regions. Observations of the core plasma potential and density are limited to ion flows 


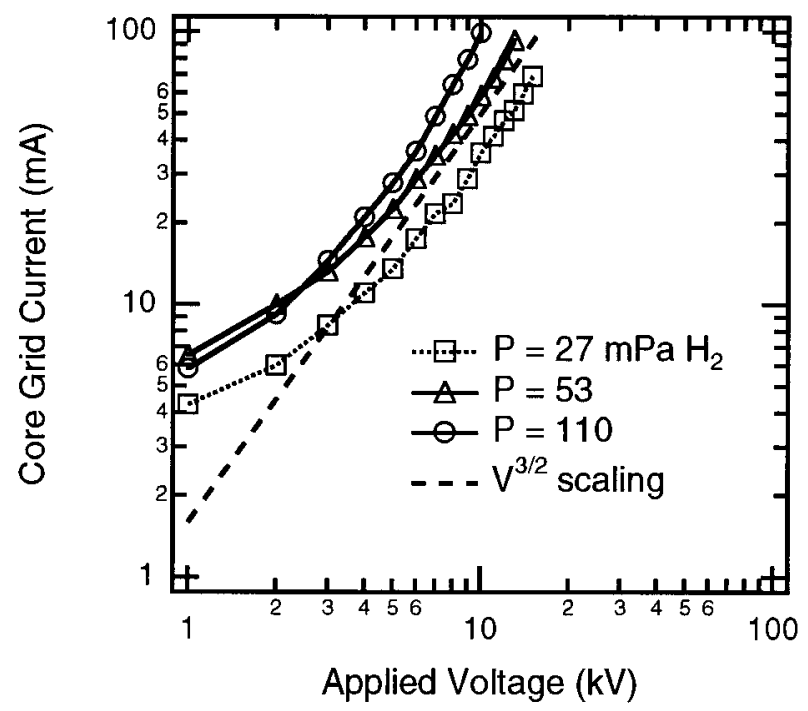

FIG. 5. Cathode current vs applied voltage for different converged core discharges showing a $V^{3 / 2}$ dependence above $3 \mathrm{kV}$ for each pressure range.

with energies $\leqslant 5 \mathrm{kV}$ to avoid thermal destruction of the probes by the energetic ions.

The perturbation of the ion flow is a concern using this invasive diagnostic technique, but no appreciable change in the discharge characteristics (i.e., cathode voltage, current, or visible core size) is observed during the measurements. In order to minimize the disturbance of the ion flow, the probe tips only intercept $\leqslant 10 \%$ of the core surface area.

\section{EXPERIMENTAL OBSERVATIONS}

Better models of system behavior and extrapolations of reactivity for higher voltages and currents can be developed by observing the effects of neutral pressure, asymmetries, injected current, and applied voltage on the ion velocity and density distributions. Hence, the WISCIF experiments discussed here concentrate on convergence scaling and measurements of the potential and density distributions throughout the ion flow, initially at relatively low energies where probe technology can be deployed.

\section{A. Space-charge-limited flow}

If the ion flow is sufficiently collisionless, then a spacecharge-limited flow into the high-voltage cathode would be expected. Indeed, measurements of the cathode current versus voltage (shown in Fig. 5) feature the $V^{3 / 2}$ scaling consistent with Eq. (5) for voltages above $3 \mathrm{kV}$ in a variety of low pressure cases. The increase observed in $I_{\text {cat }}$ with higher pressures is indicative of the increased edge plasma density with pressure (plasma source biases were held constant).

The measured radial plasma potential distributions show detailed agreement with the space-charge potential model in the mantle region [Eq. (6)], plus a variety of phenomena inside the central cathode grid. Figure 6 shows plots of the plasma potential, measured via an emissive probe using the inflection method, ${ }^{22}$ and the probe floating potential as a function of radius for a typical low pressure case $(P=13$ $\left.\mathrm{mPa} \mathrm{H}_{2}, V_{\text {cat }}=5.0 \mathrm{kV}, I_{\text {cat }}=40 \mathrm{~mA}\right)$. For these detailed probe

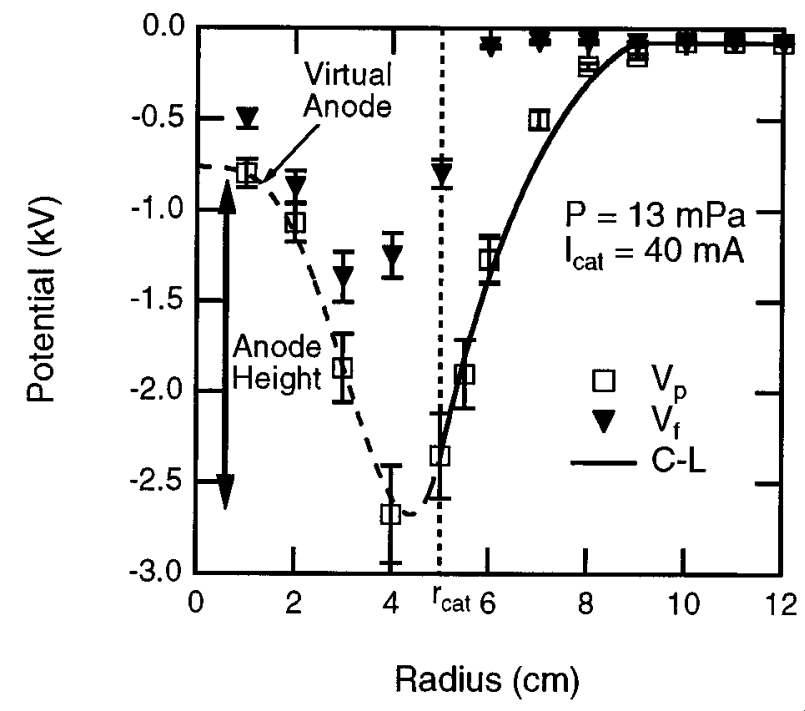

FIG. 6. Radial profile of the floating $\left(V_{f}\right)$ and plasma $\left(V_{p}\right)$ potentials for a $13 \mathrm{mPa}, 5.0 \mathrm{kV}, 40 \mathrm{~mA}$ case. Also included (solid line) is a normalized fit of the Child-Langmuir $(C-L)$ potential distribution as expected from Eq. (6) (for $r>r_{\text {cat }}$ ).

scans, ion flows with energies up to $5.0 \mathrm{kV}$ in either 13 or 27 $\mathrm{mPa}$ of hydrogen fill pressure were studied, and the collected cathode currents at these conditions were either 20 or $40 \mathrm{~mA}$.

A fit of Eq. (6) normalized to the observed voltage at $r=5.0 \mathrm{~cm}$ and using an anode radius of $9.0 \mathrm{~cm}$ shows good agreement with the measured plasma potential profile, as seen in Fig. 6. The theoretical current limit for the included best-fit graph in Fig. 6 is $150 \mathrm{~mA}$, which is 7.5 times greater than the collected ion current of $20 \mathrm{~mA}$ (assuming $\delta \approx 1$ ). ${ }^{23}$ This roughly agrees with the geometrical recirculation factor of 8.6 calculated from Eq. (7) for the globe used $(\eta=0.94)$.

Figure 7 includes comparisons of the radial potential profile against different currents and pressures, and the space-charge-limited model fits the measured data well in the mantle region. The amount of recirculating current determined as above also remains fairly constant for the three different cases studied (see Table I). The effective source radius and the voltage at $r=5.0 \mathrm{~cm}$ appear to selfconsistently adjust to provide the required space-chargelimited current density.

The observed potential at $5.0 \mathrm{~cm}$ is not equal to the applied $5.0 \mathrm{kV}$ due to Debye shielding of the grid wires $\left(\lambda_{\mathrm{D}}\right.$ is typically measured $\sim 0.05 \mathrm{~cm}$ ) and to asymmetries in the potential because of the finite wire spacing. The measurements shown in Fig. 6 are from between cathode wires, where the plasma potential is weakest. As much as a factor of 2 difference between the applied cathode voltage and the observed potential between grid wires is observed.

For neutral Maxwellian plasmas, the floating potential $\left(V_{f}\right)$ of a probe is normally biased negative with respect to the plasma potential due to the higher mobility of electrons to reach the probe surface $\left(I_{i}=I_{e}\right.$ at $\left.V_{f}\right)$. A comparison of the measured floating and plasma $\left(V_{p}\right)$ potentials in the mantle and plateau regions of a low pressure SCIF (see Fig. 6) indicates the probe biases itself positive at the floating potential $\left(V_{f}>V_{p}\right)$ to reflect the direct ion flow and collect the 

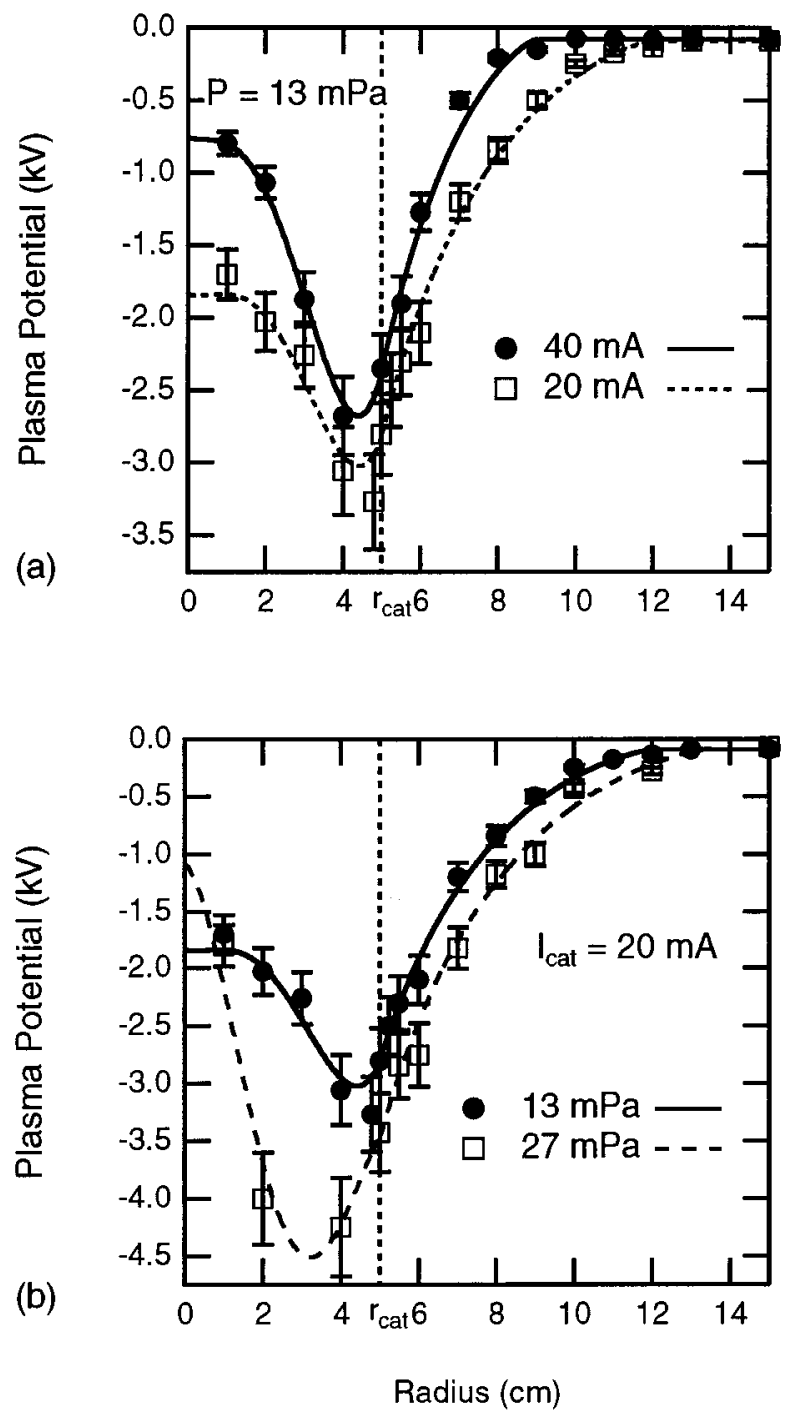

FIG. 7. Plasma potential measurements for different (a) cathode currents and (b) neutral background pressures. Included is the normalized ChildLangmuir potential distributions for $r \geqslant r_{\text {cat }}$ and spline fits through the data for $r<r_{\text {cat }}$.

local electron saturation current. This signifies a deficiency of electron current in the ion flow, and hence $I_{i}>I_{e}$ for these low pressure conditions. The difference between $V_{f}$ and $V_{p}$ is not as pronounced in the central core region, especially for higher pressure cases, which suggests a higher electron density or temperature in the core.

Figures 6 and 7 also show a virtual anode (i.e., potential hill) in the converged core region where the ion charge den-

TABLE I. Theoretical currents [from Eq. (5)] and the estimated recirculation factor for the three cases shown in Fig. 7 (applied cathode voltage $=-5000 \mathrm{~V})$.

\begin{tabular}{cccccc}
\hline \hline $\begin{array}{c}\text { Pressure } \\
(\mathrm{mPa})\end{array}$ & $\begin{array}{c}\text { Cathode grid } \\
\text { current } \\
(\mathrm{mA})\end{array}$ & $\begin{array}{c}\text { Eff. source } \\
\text { radius } \\
(\mathrm{cm})\end{array}$ & $\begin{array}{c}\text { Eff. cathode } \\
\text { voltage } \\
(\mathrm{V})\end{array}$ & $\begin{array}{c}\text { Theoretical } \\
\text { current } \\
(\mathrm{mA})\end{array}$ & $\begin{array}{c}\text { Estimated } \\
\text { recirculation } \\
\text { factor }\end{array}$ \\
\hline 13 & 20 & 12.0 & -2700 & 71 & 7.1 \\
13 & 40 & 9.0 & -2300 & 150 & 7.5 \\
27 & 20 & 13.5 & -3400 & 71 & 7.1 \\
\hline \hline
\end{tabular}

sity increases due to radial convergence. A complete virtual anode $[V(r \approx 0)=0]$ would be expected for the case of a perfectly converging ion flow with no electrons. Under all conditions studied, the core virtual anode is a simple monotonically decreasing potential hill as $r$ increases from 0 to $r_{\text {cat }}$. This structure agrees qualitatively with past experimental findings ${ }^{3}$ and particle-in-cell (PIC) simulations that account for perpendicular flow velocities from a finite velocity spread in the edge region. ${ }^{24}$

However, the simple, purely radial flow model breaks down near the origin as the impact of perpendicular velocities becomes significant, and the ion velocity distribution approaches a spherical shell in velocity space in the core region [as shown in Fig. 2(d)]. This effect is seen experimentally with double-probe $I-V$ characteristics from the plateau and core regions. Since $I_{i}>I_{e}$ in the ion flow, the double probe actually samples the energetic ion beam distribution instead of the electron distribution as in normal, nonflowing Maxwellian plasmas. The slope of the $I-V$ trace near $V=0$ is then related to the spread of the ion energy distribution, where a beam-like distribution leads to a large slope at $V=0$. Figure 8 includes three $I-V$ characteristics taken in the plateau and core regions, and it can be seen that the slope decreases as the probe enters the core region. The sharp slope seen in the plateau region is indicative of a low-temperature or beam-like distribution, which is similar to the ideal distribution expected in that region [see Fig. 2(c)]. Similarly, the broader slope in the core region is consistent with a broad energy or isotropic distribution, which resembles the ideal spherical-shell distribution expected in the core region and shown in Fig. 2(d). The detailed structure of the ion energy distribution in the core region cannot be well determined using this measurement technique, but an obvious change in the character of the ion distribution is seen between the plateau and core regions.

\section{B. Flow convergence}

The reactivity of a beam-beam dominated SCIF device is inversely proportional to the convergence radius of the ion flow, ${ }^{9}$ and it is important to understand and characterize any system parameters (i.e., accelerating well symmetry, applied voltage, injected current, neutral pressure) that may limit the flow convergence. For example, beam-beam reactivity is expected to scale with the injected current squared, but, if the core size also gets larger with current, the reaction rate will then not increase quadratically.

Several measurements indicate that the converged ion core size reduces, and hence the flow convergence is improved, as the overall current is lowered, the neutral pressure is raised, or the accelerating voltage is increased. These results are seen in Figs. 7 and 9, which show the potential structures in the core region (Fig. 7) and the half-width at half maximum (HWHM) radius of the core intensity profiles from the CCD images (Fig. 9). Figure 7(a) indicates the core anode height increasing with $I_{\text {cat }}$ while Fig. 9(a) shows an increase in HWHM with $I_{\text {cat }}$. Figures 7(b) and 9(b) indicate a strong decrease of the core size with neutral gas pressure $(P)$, and Fig. 9(b) also indicates a decrease of the core size with applied voltage $\left(V_{\text {cat }}\right)$. 
Plateau

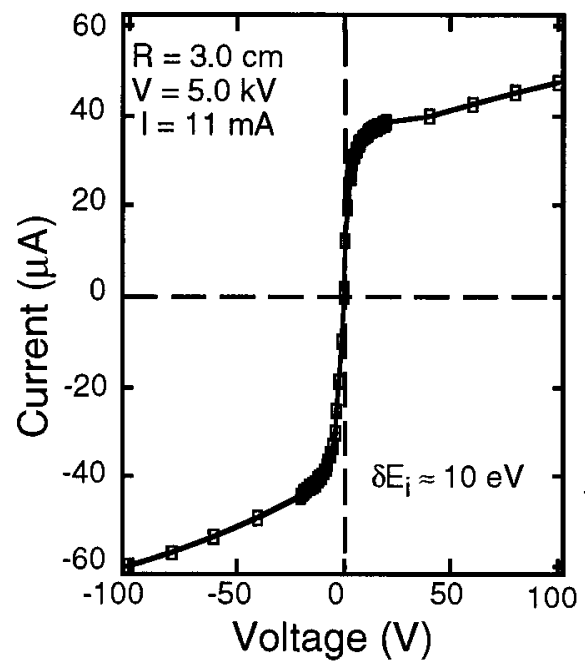

Edge of core

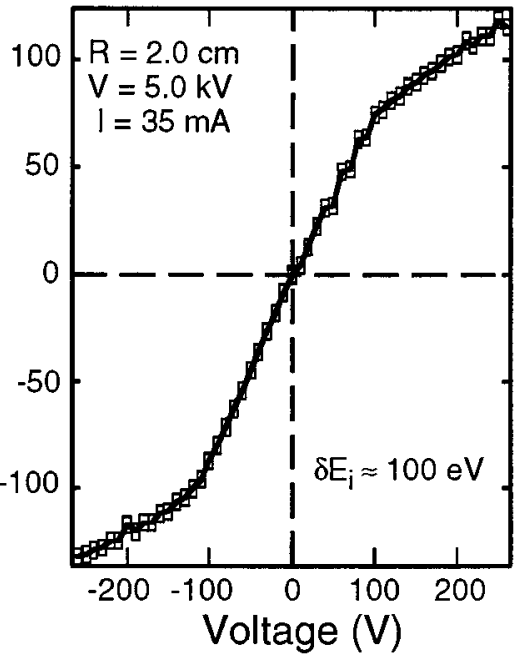

Core

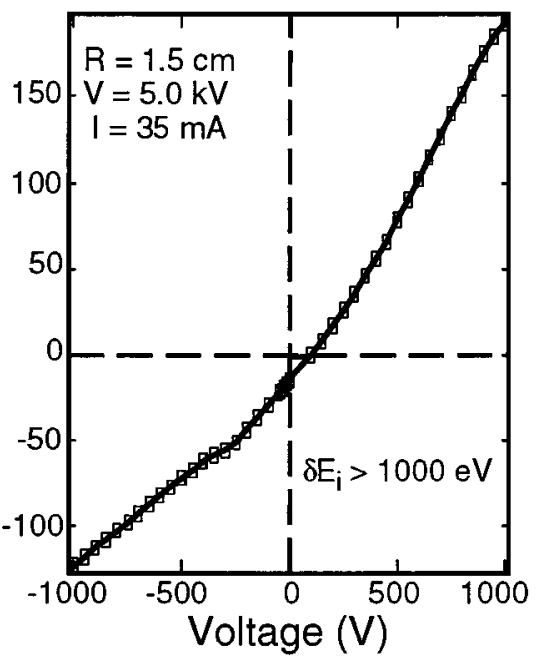

FIG. 8. Double-probe $I$ - $V$ characteristics for different radii near and inside the converged core region.

For comparison, the ideal geometric limit given by Eq. (1) is also included in Fig. 9(a). In all cases, the observed core size is larger than the ideal limit, but the tightest cores of $0.6 \mathrm{~cm}$ radius are within a factor of $4-5$ of the limit for those conditions $\left(V_{\text {cat }}=10 \mathrm{kV}, \Phi_{c} \sim 5 \mathrm{kV}, T_{i \perp} \sim 0.1 \mathrm{eV}\right.$, $R=20 \mathrm{~cm})^{25}$
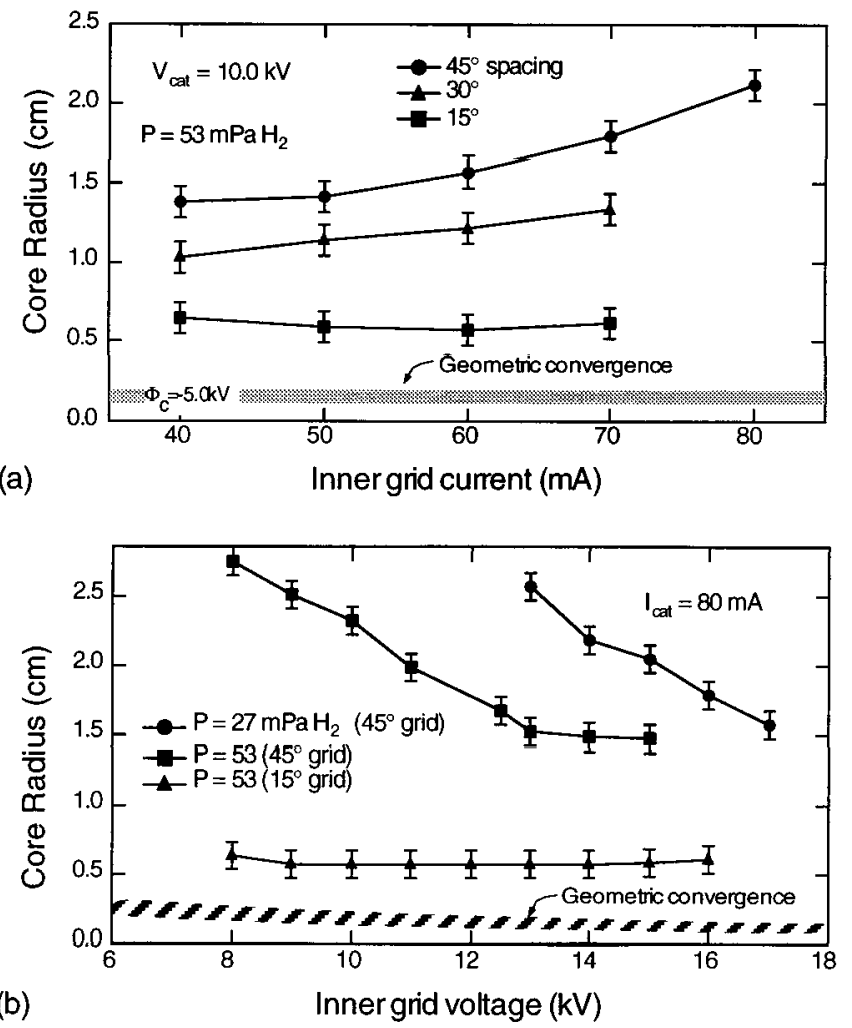

FIG. 9. Core size measurements for $45^{\circ}, 30^{\circ}$, and $15^{\circ}$ poloidal spacing grids as derived from the HWHM of the visible $\left(\mathrm{H}_{\alpha}\right)$ core emission for variations of (a) cathode current and (b) cathode voltage. Included is the estimated geometric convergence estimate from Eq. (1).
The decrease observed in $r_{c}$ with $V_{\text {cat }}$ is much faster than the inverse square root scaling expected from Eq. (1), and the most likely cause of this nonideal convergence behavior is asymmetries in the electrostatic potential from the finite grid wires of the cathode. The potential near the cathode grid wires is more negative than its value between grid wires (as discussed above in Sec. IV A), and this results in a significant perpendicular force on the ions toward the wires. In addition, the region of highest acceleration for the flow is at radii nearest the cathode, where these large irregularities in the potential are also found. The magnitude of these potential variations decreases as the grid wire spacing decreases, and therefore finely spaced grids (e.g., $15^{\circ}$ poloidal spacing) would be expected to yield smaller core sizes than more coarsely spaced $\left(45^{\circ}\right.$ spacing) grids.

Indeed, the observed core sizes decrease with decreasing grid spacing, and Fig. 9 includes comparisons of the core size scalings for differently spaced grids. Intensity profiles through the core region for the $45^{\circ}$ and $15^{\circ}$ poloidal spacing grids are shown in Fig. 10, which perhaps most dramatically shows the dependence of core size on potential well asymmetries. Here, a factor of 2.5 reduction in core size results from tripling the density of grid wires on the cathode and holding all other parameters fixed. Note that by increasing the grid wire density, the grid transparency, and hence the amount of recirculating current, decreases. The measured HWHM tends to decrease with decreasing current, but this effect is not strong enough to account for the factor of 2.5 observed. Therefore, the observed enhancement of convergence is mainly due to the improved symmetry of the accelerating potential well.

By rotating the cathode globe past a fixed emissive probe, the poloidal variations in the plasma potential are measured. Figure 11 shows the angular variation of the plasma potential for data taken at $r=5.0,5.5$, and $8.0 \mathrm{~cm}$ for the $53 \mathrm{mPa}, I_{\text {meas }}=20 \mathrm{~mA}$ case. For comparison, the expected vacuum-field variations due to the longitudinal grid 

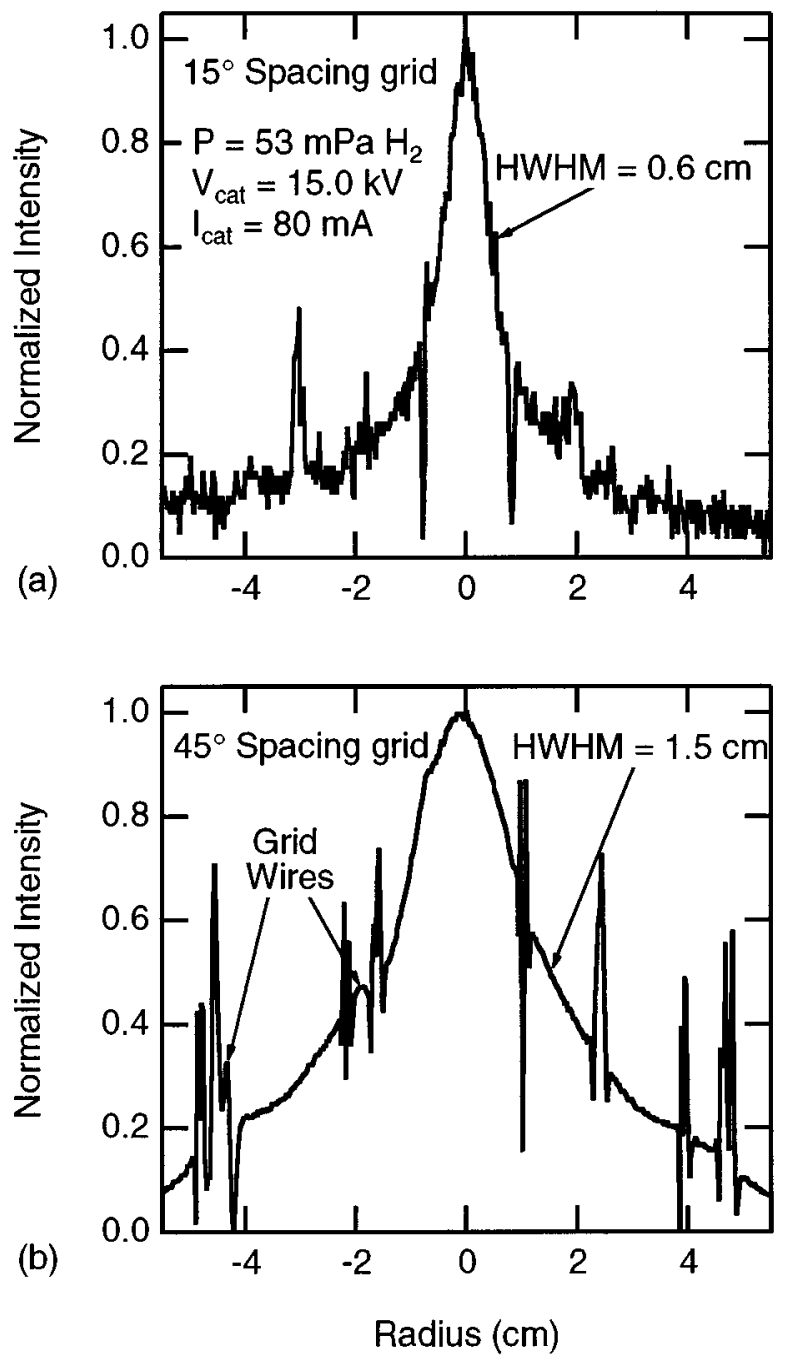

FIG. 10. Visible emission intensity profiles for the (a) $45^{\circ}$ and (b) $15^{\circ}$ poloidal spacing grids.

loops are included using superposition of the potential distribution of a single loop, given by ${ }^{26}$

$$
\Phi(r, \theta)=q \sum_{l=0}^{\infty}\left(\frac{r_{<}^{l}}{r_{>}^{l+1}}\right) P_{l}(0) P_{l}(\cos \theta),
$$

where $P_{l}$ is a Legendre polynomial, and $r_{<}$and $r_{>}$are the lesser/greater of the field and loop radii. The corresponding vacuum potential calculations from Eq. (9) are also indicated using the value of $q$ that best fits the data for each of the three different radii. These normalized vacuum and measured plasma potentials match very well for the conditions shown in Fig. 11, which is generally true for all cases investigated.

The discrepancy near the wires is due to local Debye shielding which is not accounted for in Eq. (9) above. The data at $r=5.0 \mathrm{~cm}$ hence indicates a shielding of $\approx 400 \mathrm{~V}$, and the amount of shielding appears to strongly depend on the grid current and background neutral density. This is shown in Table I through the dependence of the effective cathode voltage ( $\left.V_{\text {eff }}\right)$ on pressure and current (less negative $V_{\text {eff }}$ represents larger shielding). Qualitatively, a larger amount of shielding near the wires results in poorer flow convergence,

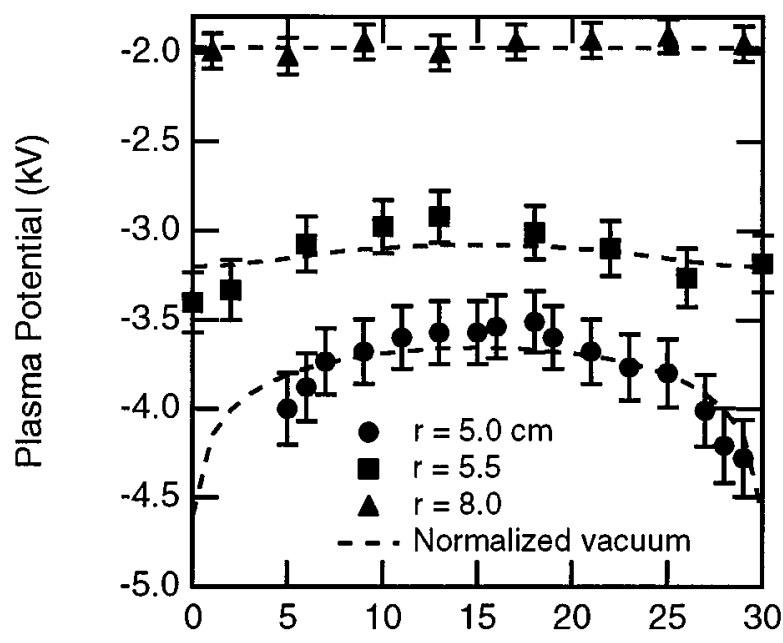

Poloidal angle $\left(^{\circ}\right)$

FIG. 11. Plasma potential measurements vs poloidal angle for a $5.0 \mathrm{kV}, 20$ $\mathrm{mA}, 53 \mathrm{mPa}$ ion flow at radii of $5.0,5.5$, and $8.0 \mathrm{~cm}$. Grid wires are spaced at $0^{\circ}$ and $30^{\circ}$ for this cathode.

for ions will have less energy entering the core region and the larger potential gradients near the wires will scatter the ions further. This effect therefore contributes to and may account for the observed core size scalings with pressure and current.

\section{Core density}

Measurements of the core density employ a scannable floating double probe. However, shadowing of the recirculating ion flow by the radial alumina probe shaft leads to an underestimation of the ion density, and the measured electron saturation current, $i_{\text {sat }}$, is therefore corrected by the recirculation factor, $\xi$ [from Eq. (7)], to account for the shadowing effect and provide an upper limit for the ion density from these measurements (in this case, $\eta \approx 0.91$ and $\xi=5.8$ ). Since $V_{f} \sim V_{p}$ in the core, the ion current $\left(I_{i}=e n_{i} v_{i} A_{p}\right)$ approximately equals $i_{\text {sat }}$, and the ion density is then given by

$$
n_{i}=\frac{\xi i_{\text {sat }}}{e A_{p}} \sqrt{\frac{M}{2 q \Phi_{p}}}
$$

where $A_{p}$ is the probe area $\left(=5.9 \times 10^{-6} \mathrm{~m}^{2}\right)$. For the core in Fig. $8, i_{\text {sat }} \geqslant 150 \mu \mathrm{A}$ and $\Phi_{p}=2500 \mathrm{~V}$, giving the ion density calculated from Eq. (10) to be $\geqslant 1.3 \times 10^{15} \mathrm{~m}^{-3}$.

Another approximation of the core density is given by the measured current collected at the cathode grid. For a gridded system, the effective cathode transparency $(\eta)$ leads to a fractional loss of the current between the mantle $\left(I_{\operatorname{man}}\right)$ and core $\left(I_{c}\right)$ regions, and hence, $I_{c}=\eta I_{\operatorname{man}}$. Using the definition of the recirculation factor [Eqs. (7) and (8)], $I_{c}$ can be related to the measured grid current $\left(I_{\text {meas }}\right)$ by

$$
I_{c}=\eta I_{\text {man }}=\frac{\eta \xi I_{\text {meas }}}{(1+\delta)}=\frac{\eta I_{\text {meas }}}{\left(1-\eta^{2}\right)(1+\delta)},
$$


TABLE II. Core ion density measurements and calculations $\left(\mathrm{m}^{-3}\right)$ for a 5 $\mathrm{kV}, 35 \mathrm{~mA}$ ion flow at $53 \mathrm{mPa}$.

\begin{tabular}{cccc}
\hline \hline $\begin{array}{l}\text { Double probe } \\
\text { measurement } \\
\text { Eq. }(10)\end{array}$ & $\begin{array}{c}\text { Cathode current } \\
\text { estimate }\end{array}$ & $\begin{array}{c}\text { Pyrometric } \\
\text { estimate }\end{array}$ & $\begin{array}{c}\text { Simple flow model } \\
\text { estimate }\end{array}$ \\
\hline $1.3 \times 10^{15}$ & Eq. (12) & Eq. (13) & Eq. (3) \\
\hline \hline
\end{tabular}

which can also be related to the core density since $I_{c}=e n_{\mathrm{ic}} v_{c} \pi r_{c}^{2}$. Therefore, the expected core density for a particular grid current is

$$
n_{\mathrm{ic}}=\frac{\eta I_{\text {meas }}}{\left(1-\eta^{2}\right)(1+\delta)}\left(\frac{1}{e v_{c} \pi r_{c}^{2}}\right) \text {. }
$$

Again assuming $\delta \approx 1$ and $\Phi_{c} \approx 2500 \mathrm{~V}$ for these conditions ( $r_{c}=1.6 \mathrm{~cm}$ for this $5 \mathrm{kV}, 35 \mathrm{~mA}$ discharge), Eq. (12) gives a core density of $1.0 \times 10^{15} \mathrm{~m}^{-3}$.

Another estimate of the core density is provided by pyrometric measurements of a single alumina probe tip inserted into a higher energy core. The tip temperature can reach over $2200{ }^{\circ} \mathrm{C}$ for a $10 \mathrm{kV}, 80 \mathrm{~mA}$ discharge and, assuming that the power deposited is solely radiated away, then the ion density can be calculated from power balance on the probe tip

$$
n_{i} \approx \frac{P_{\mathrm{rad}}}{v_{i} E_{i}}=\frac{\epsilon \sigma T_{\mathrm{probe}}^{4}}{\sqrt{2 / M}\left(q \Phi_{c}\right)^{3 / 2}},
$$

where $\epsilon$ is the emissivity of the alumina $(\approx 0.7),{ }^{27} \sigma$ is the Stefan-Boltzmann constant, and $T_{\text {probe }}$ is the surface temperature of the probe. For the $10 \mathrm{kV}$ case, where $q \Phi_{c} \approx 5000$ $\mathrm{eV}$ and $T_{\text {probe }} \approx 2500 \mathrm{~K}, n_{i c}$ is approximately $1.9 \times 10^{15} \mathrm{~m}^{-3}$. For these conditions, Eq. (12) also predicts a core ion density of $1.5 \times 10^{15} \mathrm{~m}^{-3}\left(r_{c} \approx 2.1 \mathrm{~cm}\right.$ for $10 \mathrm{kV}, 80 \mathrm{~mA} ; 45^{\circ}$ spacing grid, $\eta=0.94$ ), which is in good agreement with the pyrometric estimate.

A fourth estimate of the core ion density is given by the simple flow model [from Eq. (3)]. The edge density $(r=20$ $\mathrm{cm}$ ) was measured with a standard Langmuir probe to be $2.2 \times 10^{14} \mathrm{~m}^{-3}$ for the $5 \mathrm{kV}, 35 \mathrm{~mA}$ case described above, but the value of $E_{\|}$at $20 \mathrm{~cm}$ needed for Eq. (3) is yet undetermined. If the edge plasma behaves as a uniform plasma, then the ions will fall into the accelerating potential boundary at the local Bohm velocity [or $E_{\|}(R)=0.5 T_{e} \approx 5 \mathrm{eV}$ ]. However, since the edge plasma has different boundary conditions (biased grids) than a typical uniform plasma, it is not clear the ions actually will enter the accelerating region at the Bohm speed. A minimal estimate is given by the ion thermal velocity $\left[\right.$ or $\left.E_{\|}(R) \sim 0.1 \mathrm{eV}\right]$. Therefore the predicted core density from Eq. (3) is $6.1 \times 10^{15} \mathrm{~m}^{-3}$ if $E_{\|}(R)=5 \mathrm{eV}$ or $0.9 \times 10^{15}$ $\mathrm{m}^{-3}$ if $E_{\|}(R)=0.1 \mathrm{eV}$ (again using $r_{c}=1.6 \mathrm{~cm}, \Phi_{c} \approx 2500$ $\mathrm{V})$.

These varied core density measurements and calculations are shown in Table II, with the pyrometric estimate scaled to the conditions of the double-probe measurements using Eq. (12). These values of the core ion density indicate an overall increase in density by a factor of 5-10 compared to the edge plasma.
In general, the increase in core density, compared to the cold edge density, is in relatively good agreement with the simple estimate given by Eq. (3), and hence the ion density appears to follow classical expectations at the conditions studied.

\section{DISCUSSION AND CONCLUSIONS}

The plasma potential measurements for the low pressure ion flows discussed above are consistent with a collisionless, space-charge-limited flow model in the mantle region. In addition, both the effective source radius $\left(R_{\text {eff }}\right)$ and cathode voltage $\left(V_{\text {eff }}\right)$ adjust accordingly to provide the required current density for the edge conditions. Table I shows that, as expected from the space-charge model, $R_{\text {eff }}$ decreases (through $\alpha$ ) as $I_{\text {cat }}$ increases (at constant $V_{\text {cat }}$ and $P$ ), but note that $V_{\text {eff }}$ also decreases. This is attributed to enhanced shielding of the cathode grid wires by the increased local plasma density at higher currents. The increase in $V_{\text {eff }}$ and $R_{\text {eff }}$ for increased pressure (at constant $V_{\text {cat }}$ and $I_{\text {cat }}$ ) is also consistent with the space-charge model, for $R_{\text {eff }}$ increases to offset the increased edge plasma density and $V_{\text {eff }}$ observed at higher pressure.

The symmetry and depth of the accelerating potential well improve with increasing cathode voltage and pressure and with decreasing current and grid wire spacing, which results in improved ion flow convergence (cf. Figs. 9 and 10). The decrease in core size with voltage is much more rapid than the inverse-square-root expectation from Eq. (1). In addition, the data shown in Fig. 9(b) suggest that the converged core size may saturate at a level still 4-5 times higher than the geometric convergence expectation from Eq. (1). Hence, Eq. (1) does not adequately predict the ion flow convergence due to the large asymmetries in the accelerating potential well, and the expected core density scaling from Eq. (4) would be invalid as well.

To better estimate the expected core size in the presence of the observed potential variations, a Monte Carlo simulation of single ion orbits in an asymmetric potential well is used. For these calculations, a two-dimensional model of the plasma potential is generated using a space-charge-limited distribution in the mantle region and a Gaussian-shaped virtual anode inside the cathode grid. The magnitude of the asymmetries in the model is determined using the variations in the vacuum fields given by the superposition of the fields generated by the longitudinal loops of the cathode grid [as described in Sec. IV B, Eq. (9)]. The model potential also takes into account Debye shielding of the cathode near the grid wires.

The average radius of closest approach, $\left\langle r_{i}\right\rangle$, of 2500 test particles estimates the converged core size and is defined for each transit $i$ of the ions through the core region. The resulting average core size, $\left\langle r_{c}\right\rangle$, is then obtained by averaging over all transits weighted by the fraction of particles making the particular transit $\left(N_{i}\right)$

$$
\left\langle r_{c}\right\rangle=\frac{\sum_{i} N_{i}\left\langle r_{i}\right\rangle}{\sum_{i} N_{i}}
$$




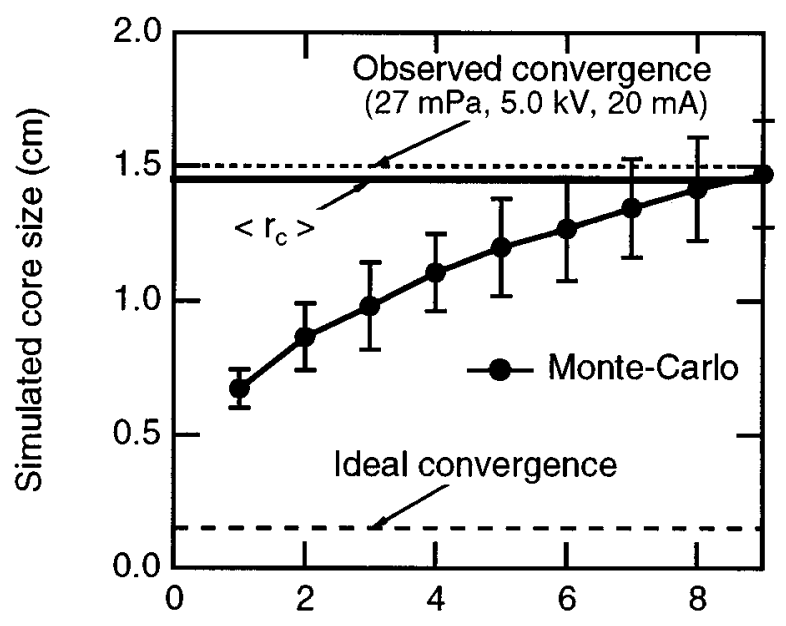

Number of Transits

FIG. 12. Core size estimates vs the number of transits through the core region derived from Monte Carlo simulations of ion trajectories in a twodimensional asymmetric well. Included are the ideal convergence estimate and the observed core size for the $27 \mathrm{mPa}, 5.0 \mathrm{kV}, 20 \mathrm{~mA}$ ion flow.

where $i$ is the transit number index. The results of such calculations for the $27 \mathrm{mPa}, 5.0 \mathrm{kV}, 20 \mathrm{~mA}$ case studied in Sec. IV are shown in Fig. 12. The values of $\left\langle r_{i}\right\rangle$ for the test ion population are plotted as a function of the number of transits through the core, and for comparison, the ideal geometric core size, the measured core radius, and $\left\langle r_{c}\right\rangle$ are also included in Fig. 12.

As expected, the average core size increases with the number of transits, confirming that the grid asymmetries rapidly defocus the ion trajectories. The convergence also rapidly degrades from the ideal convergence expectation as the number of passes through the system increases. In addition, $\left\langle r_{i}\right\rangle$ approaches the measured core size as the number of transits through the core region approaches the experimental recirculation factor $(\xi=8.6)$. In all cases studied, the simulation predicts either a smaller or equivalent core size compared to experiment, and therefore this single particle orbit estimate is consistent with the measured core size, given the observed equilibrium potential structure in the core region and the asymmetries in the accelerating potential well.

To further confirm the effect of the accelerating potential well symmetry on convergence, the results of Monte Carlo simulations for three different cathode grid wire spacings are shown in Fig. 13. The simulations predict smaller core sizes with reduced grid spacing, which is also observed experimentally [cf. Fig. 9(a)].

However, the experiments using the $15^{\circ}, 30^{\circ}$, and $45^{\circ}$ grid spacings do not keep the grid transparency constant $(\eta$ $=0.85,0.91$, and 0.94 , respectively), and the resulting change in the recirculation factor also influences the flow convergence by changing the average number of transits an ion makes through the asymmetric fields. The simulation then predicts that $\left\langle r_{c}\right\rangle=0.6,1.0$, and $1.4 \mathrm{~cm}$ for the $15^{\circ}, 30^{\circ}$, and $45^{\circ}$ spacing grids, respectively (see Fig. 13), which is consistent with the variations seen experimentally [cf. Fig. $9(\mathrm{a})]$.

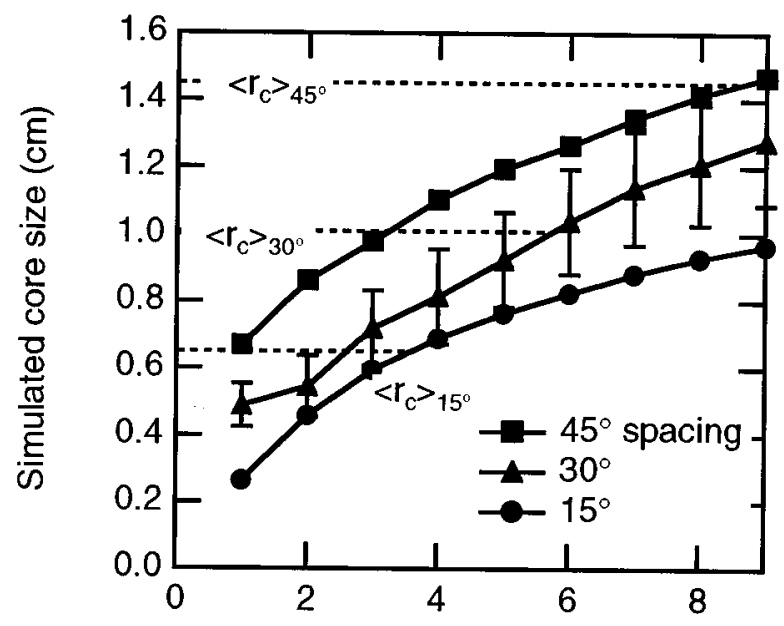

Number of Transits

FIG. 13. Core size results calculated from the Monte Carlo ion trajectory simulations for $15^{\circ}, 30^{\circ}$, and $45^{\circ}$ cathode grid spacings. Included are the estimates of $\left\langle r_{c}\right\rangle$ calculated from Eq. (14) using the experimental cathode transparencies for determining $N_{i}$.

The simulation results in Fig. 13 suggest that each of these effects contributes equally to the predicted core size variations. Therefore, it is the combination of the improved well symmetry with decreasing grid spacing and the reduced recirculation factor from the decreased grid transparency that accounts for the observed dependence of the flow convergence with the cathode geometry.

This collisionless model neglects the effects from neutral interactions, but a significant decrease in core radius is observed with increasing neutral pressure [cf. Fig. 9(b)]. Emissive probe measurements indicate that the depth and symmetry of the accelerating potential well improve with increasing pressure. This alone may account for the observed improvement in convergence, but ion-neutral collisions may also affect the overall convergence.

For example, if ion-neutral collisions remove a fraction of the recirculating ions from the system (e.g., via chargeexchange), then the effective number of transits through the asymmetric fields would be reduced, and the Monte Carlo simulations suggest that a smaller core size would then be expected (see Fig. 12). The effective system transparency against charge-exchange collisions, defined as the percentage of the ion flow that does not charge-exchange on its transit to the core, ranges from $0.97-0.95$ for the experimental conditions studied $(13-27 \mathrm{mPa}){ }^{18}$ This indicates that, although the ions are primarily lost to the cathode grid $(\eta=0.94)$, charge-exchange collisions could significantly reduce the amount of recirculating ion current, and smaller converged core sizes would then result as the background pressure increases. However, the estimated recirculation factor stays relatively constant between the 13 and $27 \mathrm{mPa}, 20 \mathrm{~mA}$ cases (see Table I), which suggests that charge-exchange does not reduce the overall system transparency for these low pressure conditions.

It is also possible that after gaining significant angular momentum from the asymmetries in the accelerating well, 
ions may then collide with neutrals at the edge radial turning points. This would help thermalize the ion distribution there, and the resultant reduction of the ion angular momentum allows each transit of the ion through the core to appear as an undisturbed first transit. The experimental edge collisional mean-free-paths are consistent with this argument for the pressure ranges studied $(\lambda \approx 25-50 \mathrm{~cm}) .{ }^{18}$ However, further experimental evidence (e.g., measurements of the perpendicular ion temperature versus pressure) is needed to confirm the relevance of these neutral collisional effects.

In present gridded systems, convergence is not important since beam-target fusion reactions dominate the reactivity of these devices, as evidenced by the linear scaling of reactivity with the cathode current. ${ }^{1-3}$ In fact, convergence may reduce the reactivity by forming a virtual anode that reduces the energy, hence decreasing the probability for fusion, of the ions at the center. However, good convergence is required to achieve optimal beam-beam reactivity scaling for the applications that require higher fusion reaction rates, and the importance of symmetry in determining convergence places a constraint on any SCIF device planned for these applications. The observed loss of convergence with decreasing pressure and increasing current makes achieving significant beambeam scaling far less favorable.

A single virtual anode is measured by the emissive probe in the core region, but no evidence of multiple-well structures is observed in the WISCIF experiments under the iondominated, low pressure conditions discussed here. Some computer simulations, performed by other investigators, show multiple-well formation, but these models assume perfectly symmetric accelerating potentials. ${ }^{1,13,14}$ However, the experiments indicate that any detailed potential structure near $r=0$ would be washed out due to the angular momentum of the ions gained from the asymmetries in the accelerating region.

Presumably, the virtual anode near $r=0$ can act as an efficient trap for warm electrons, and it may allow the trapped electrons to be heated to temperatures on the order of the anode height (shown in Fig. 6 as the difference between the potential at $r=0$ and the most negative potential inside the cathode) via electron-ion collisions. However, the measured probe floating potentials are more positive than the plasma potential, which signifies that the local ion current to the probe is larger than the electron current at the plasma potential. Any hot electron population must be of much lower density than the local ion density for $I_{e}$ to remain less than $I_{i}$ at the plasma potential.

While these experiments were carried out at lower voltages and densities than needed for applications, these results at low pressure indicate that the generic ion flow behavior is essentially collisionless and classical at the conditions studied. In order to study the more application-relevant ion energies and densities, new techniques will need to be developed to diagnose the higher energy density systems.

\section{SUMMARY}

These results represent, to the best of our knowledge, the first detailed evaluation of the ion flow characteristics in the low-pressure, converged-core regime of a spherically con- vergent ion focus. Measurements of the electrostatic potential distribution throughout the WISCIF device show detailed agreement with a recirculating space-charge-limited flow model and indicate the ions dominate the system at these low pressures $(<53 \mathrm{mPa})$. A virtual anode structure forms in the converged core, due to the enhanced ion density in that region, but no evidence of multiple potential well structures is seen for the conditions under study. Camera imaging and plasma potential measurements in the core region show that flow convergence improves with increasing voltage, pressure, potential well symmetry, and decreased current. The tightest observed core sizes $\left(r_{c}=0.6 \mathrm{~cm}\right)$ are within a factor of $4-5$ of the ideal geometric limit given by Eq. (1) and are consistent with a collisionless multipass orbit model prediction given the degree of asymmetries measured in the accelerating potential well. Core density measurements show a factor of $\sim 10$ increase in ion density in the core region, which agrees with a simple flow conservation model.

Understanding the influence of the virtual anode on the flow dynamics is an important issue for high-density systems. The height of the anode will significantly affect the density, reactivity, and collision rates in the core, and bremsstrahlung and ion-electron collisional energy losses there will reduce the efficiency and performance of the highdensity systems. Future work will concentrate on these issues and on determining the influence of flow convergence, ionneutral collisions, and cathode voltage, current, and geometry on the system reactivity at application-relevant energies and densities (i.e., $V_{\text {cat }} \approx 75 \mathrm{kV}, n_{\text {ic }} \sim 10^{17} \mathrm{~m}^{-3}$ ) for deuterium plasmas.

\section{ACKNOWLEDGMENTS}

The authors would like to thank R. Ashley, R. Buckles, and B. Foucher for their technical assistance and N. Hershkowitz, R. Hirsch, G. Kulcinski, G. Miley, R. Nebel, W. Nevins, and J. Santarius for their useful discussions and contributions on this matter.

This work was supported, in part, by the Electric Power Research Institute under agreement No. 6424-51010 and the University of Wisconsin College of Engineering.

${ }^{1}$ R. Hirsch, J. Appl. Phys. 38, 4522 (1967).

${ }^{2}$ G. H. Miley, J. Nadler, T. Hochberg, Y. Gu, O. Barnouin, and J. Lovberg, Fusion Technol. 19, 840 (1991).

${ }^{3}$ J. H. Nadler, G. H. Miley, Y. Gu, and T. Hochberg, Fusion Technol. 20, 850 (1991).

${ }^{4}$ J. H. Nadler, G. H. Miley, Y. Gu, and T. Hochberg, Fusion Technol. 21, 1639 (1992).

${ }^{5}$ A. L. Gardner, D. M. Hatch, A. I. Y. Chan, and R. P. Evans, Ann. (N.Y.) Acad. Sci. 251, 179 (1975)

${ }^{6}$ W. C. Elmore, J. L. Tuck, and K. M. Watson, Phys. Fluids 2, 239 (1959).

${ }^{7}$ T. J. Dolan, Plasma Phys. Controlled Fusion 36, 1539 (1994).

${ }^{8}$ R. W. Bussard, Fusion Technol. 19, 273 (1991).

${ }^{9}$ N. A. Krall, Fusion Technol. 22, 42 (1992).

${ }^{10}$ D. C. Barnes, R. A. Nebel, and L. Turner, Phys. Fluids B 5, 3227 (1993).

${ }^{11}$ W. M. Nevins, Phys. Plasmas 2, 3804 (1995).

${ }^{12}$ T. H. Rider, Phys. Plasmas 2, 1853 (1995).

${ }^{13}$ B. E. Cherrington, J. T. Verdeyen, and D. A. Swanson, Ann. (N.Y.) Acad. Sci. 251, 139 (1975).

${ }^{14}$ J. T. Verdeyen, B. E. Cherrington, D. A. Swanson, and D. J. Meeker, Ann. (N.Y.) Acad. Sci. 251, 126 (1975).

${ }^{15}$ T. J. Dolan, J. T. Verdeyen, D. J. Meeker, and B. E. Cherrington, J. Appl. Phys. 43, 1590 (1972). 
${ }^{16}$ W. M. Black and J. W. Robinson, J. Appl. Phys. 45, 2497 (1974).

${ }^{17}$ W. M. Black and E. H. Klevans, J. Appl. Phys. 45, 2502 (1974).

${ }^{18}$ R. K. Janev, W. D. Langer, K. Evans, Jr., and D. E. Post, Jr., Elementary Processes in Hydrogen-Helium Plasmas (Springer-Verlag, Berlin, 1987).

${ }^{19}$ A. Anders, A Formulary for Plasma Physics (Akademie, Berlin, 1990).

${ }^{20}$ I. Langmuir and K. Blodgett, Phys. Rev. 24, 49 (1924).

${ }^{21}$ R. L. Hirsch, Phys. Fluids 11, 2486 (1968).

${ }^{22}$ N. Hershkowitz, in Plasma Diagnostics, edited by O. Auciello and D. L. Flamm (Academic, Boston, 1989), p. 176.

${ }^{23}$ The secondary electron emission coefficient, $\delta$, is estimated by comparing the values of $\delta$ for a variety of metals listed in Oak Ridge National Laboratory Report No. ORNL-6088, 1985 (unpublished). See National Techni- cal Information Service Document No. DE85008764. Copies may be ordered from the National Technical Information Service, Springfield, Virginia 22161.

${ }^{24} \mathrm{R}$. Nebel (private communication, 1994).

${ }^{25} \mathrm{~A}$ core anode of $5000 \mathrm{~V}$ is assumed and the edge perpendicular temperature is estimated to be $0.1 \mathrm{eV}$ for these calculations, which is a typical ion temperature for this type of glow discharge. B. Chapman, Glow Discharge Processes (Wiley, New York, 1980), p. 52.

${ }^{26}$ J. D. Jackson, Classical Electrodynamics (Wiley, New York, 1978), p. 93.

${ }^{27}$ Emissivity of alumina is estimated using the value for fire brick from the Handbook of Chemistry and Physics, 71st ed., edited by D. R. Lide (CRC, Boston, 1990), pp. 10-282. 